\title{
Identificação das espécies causadoras de milíases em humanos provenientes dos serviços de saúde do município de Nova Iguaçu, RJ, entre os anos de 2017 e 2018
}

\author{
Identification of myiasis-causing species collected in humans from health services of the city of Nova \\ Iguaçu, RJ, between the years 2017 and 2018
}

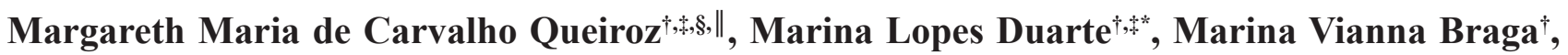 \\ Lucas Barbosa Cortinhas ${ }^{\dagger, \S}$
}

Como citar esse artigo. Queiroz,

M.M.C.; Duarte, M.L.; Braga, M.V.;

Cortinhas, L.B. Identificação das

espécies causadoras de míases em

humanos provenientes dos serviços de saúde do município de Nova Iguaçu, RJ, entre os anos de 2017 e 2018 . Revista de Saúde. 2019 Jul./Dez; 10 (2): 37-42.

\section{Resumo}

As moscas são insetos holometábolos, suas larvas são parasitas obrigatórios, alimentando-se dos tecidos vivos ou mortos do hospedeiro e merecem especial destaque em saúde pública, por produzirem miíases no homem e animais. Miíases são enfermidades comuns nas zonas rurais e periferias dos países tropicais. Este trabalho teve como objetivo identificar as espécies e instares larvais dos dípteros causadores de miíases humanas de pacientes atendidos em Hospitais Municipais (HM) e Clínicas da Saúde da Família (CSF) da cidade de Nova Iguaçu, RJ. Depois da retirada das larvas das lesões, estas foram sacrificadas em éter, lavadas em solução de $\mathrm{NaCl}$ a $0,9 \%$ e preservadas em etanol a $70 \%$. Posteriormente, foram encaminhadas ao laboratório onde foram transferidas para solução de $\mathrm{KOH} \mathrm{a} 10 \%$ e, em seguida, para lactofenol. Após este processo, foram montadas entre lâmina e lamínula, analisadas com o auxílio de microscópio estereoscópico e identificadas com o uso de chaves dicotômicas específicas. Analisou-se um total de 880 larvas, sendo 730 provenientes de atendimentos das CSF e 150 larvas dos HM. Foram identificadas três diferentes espécies, das quais Cochliomyia hominivorax foi encontrada na maioria dos casos e, Dermatobia hominis e Fannia sp. com apenas um caso cada. Nas CSF observou-se uma predominância de larvas de $3^{\circ}$ instar, resultado este corroborado pelos HM (461 e 105, respectivamente). Larvas faratas só foram identificadas nas amostras das CSF. A maior presença de $3^{\circ}$ instar (idade entre três e sete dias) indica uma possível demora na procura dos pacientes pelas instituições de saúde para o tratamento.

Palavras-chave: Biodiversidade, Diptera, Entomologia Médica, Calliphoridae, Cuterebrinae, Fanniidae.

\begin{abstract}
Flies are a holometabolous insect, and its larvae are obligatory parasites that feed on living or necrotic tissue from vertebrates hosts. The presence of these larvae on tissue is known as myiasis, a major public health disease, especially in rural and peripheral areas from tropical countries. This paper aimed to identify the species and the instar of the flies larvae collected in human patients from the Municipal Hospitals (MH) and Family Health Clinics (FHC) from the city of Nova Iguaçu, State of Rio de Janeiro. The larvae were killed in ether, washed in $\mathrm{NaCl} 0.9 \%$, and preserved in ethanol $70 \%$. Then, the samples were sent to the laboratory and transferred to a solution of $\mathrm{KOH} \mathrm{10 \% ,} \mathrm{followed} \mathrm{by} \mathrm{lactophenol.}$ After this, the samples were mounted between slide and coverslip, analyzed on a stereoscope microscope, and identified with specific dichotomic keys. For this work, we analyzed 880 larvae, 730 of which were provided by the FHC, and 150 by the MH. From these specimens, the identification of three species was made. Cochliomyia hominivorax was the most prevalent. Dermatobia hominis and Fannia sp. only have one specimen each. In the FHC sample, we could observe the predominance of third instar larvae that corroborates with the samples from the MH (461 and 105, respectively). The four pharate larvae identified were those from the CFS samples. The high prevalence of third instar larvae (age between three and seven days) suggests a delay in searching for health services for the correct treatment.
\end{abstract}

Keywords: Biodiversity, Diptera, Medical Entomology, Calliphoridae, Cuterebrinae, Fanniidae.

\section{Introdução}

Miíases são definidas como afecções ocasionadas por infestações de larvas de dípteros muscoides que utilizam hospedeiros vertebrados para o seu desenvolvimento ${ }^{1,2}$. Essas larvas podem se alimentar dos tecidos vivos, mortos, substâncias corporais ou até mesmo do alimento ingerido pelo hospedeiro, para assim, completar seu ciclo de vida até a fase adulta ${ }^{3}$.

Espécies como Cochliomyia hominivorax (Coquerel, 1858) (Diptera: Calliphoridae) e Dermatobia hominis (Linneaus Jr, 1781) (Diptera: Oestridae: Cuterebrinae) ocasionam míases obrigatórias, ou seja, as larvas são histiófagas e se desenvolvem no tecido

Afiliação dos autores: ${ }^{\dagger}$ Laboratório de Entomologia Médica e Forense (LEMEF), Instituto Oswaldo Cruz - Fundação Oswaldo Cruz (IOC/FIOCRUZ), Rio de Janeiro, RJ, Brasil;

*Programa de Pós-Graduação em Biodiversidade e Saúde, Instituto Oswaldo Cruz-Fundação Oswaldo Cruz (IOC/FIOCRUZ), Rio de Janeiro, RJ, Brasil;

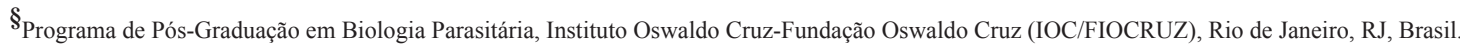

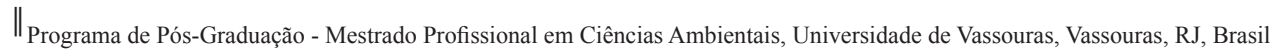

* Email de correpondência: marinaduartebio@gmail.com

Recebido em: XXXXX. Aceito em: XXXX. 
vivo, na região cutânea ou subcutânea de vertebrados (mamíferos) $^{1,2}$.

Tais espécies causam míases primárias em humanos, principalmente, em indivíduos com feridas abertas como no caso de C. hominivorax, afetando especialmente áreas de cavidades mucosas, como a região dos olhos, nariz, boca, e até mesmo ânus e genitália ${ }^{4}$.

A infestação por $C$. hominivorax, conhecida popularmente como "mosca varejeira" e causadora da chamada "bicheira", ocorre através da postura de ovos diretamentenas margens dos ferimentosnos mamíferos ${ }^{1,3}$. As larvas se alimentam dos fluidos e tecidos musculares, podendo agravar as lesões que liberarão odores atrativos a outras espécies de moscas que poderão realizar novas posturas, como por exemplo, a espécie Cochliomyia macellaria (Fabricius, 1775) (Diptera: Calliphoridae) e espécies do gênero Chrysomya (Robineau-Desvoidy, 1830) (Diptera: Calliphoridae), que podem ocasionar míases secundárias, com as larvas se alimentando do tecido necrosado ${ }^{3}$.

Dermatobia hominis ocasiona afecções em áreas não lesionadas do corpo, principalmente na região da nuca até a região lombar, especialmente em homens (por estes muitas vezes não utilizarem roupas na parte superior) ${ }^{1}$. Dermatobia hominis ("mosca-berneira") causa a chamada dermatobiose, uma miíase furuncular popularmente conhecida como "berne", que ocorre quando algum outro díptero com hábito zoofílico e hematófago, além de sinantrópico, como por exemplo, espécies das famílias Culicidae, Simuliidae, Muscidae, Sarcophagidae, Fanniidae e Calliphoridae ${ }^{1,5}$ que são capturados pelas fêmeas, que ovipõem sobre o abdômen (preferencialmente) dos insetos foréticos, que atuam como vetores mecânicos. Ao repousar em um mamífero, os ovos aderidos ao corpo são estimulados pela elevação da temperatura corporal do hospedeiro e a liberação de $\mathrm{CO} 2$, ocasionando sua eclosão ${ }^{6}$. As larvas penetram na pele, através de folículos pilosos ou de lesões tegumentares, como picadas de insetos e abrasões e se abrigam na região subcutânea, onde permanecerão para se alimentar e completar seu desenvolvimento ${ }^{7}$.

Fannia (Robineau-Desvoidy, 1830) é um gênero de mosca pertencente à família Fanniidae, na qual as larvas apresentam hábito alimentar saprófago, podendo se desenvolver tanto em fezes ou fungos ${ }^{8}$ quanto em matéria orgânica vegetal ou animal em decomposição". Por este motivo, suas larvas e adultos podem ser encontrados em ambientes com pouca higienização ou até mesmo em cadáveres, apresentando importância sanitária e forense. Espécies de Fannia também são descritas como vetores de ovos de Dermatobia hominis $^{1,10-12}$, além de serem encontradas em casos de miíases secundárias ${ }^{13}$.

Portanto, enquanto a dermatobiose não apresenta relação com hábitos higiênicos pessoais ou do ambiente, a infestação por $C$. hominivorax pode ser ocasionada por hábitos precários de higienização ou o não tratamento de feridas, estando associado em casos de pacientes com lesões diabéticas ou não; pacientes com transtornos mentais; câncer em estágio avançado; imunodeprimidos; etilista; em indivíduos com casos graves de gengivites ou outras lesões na cavidade oral e/ou outras áreas do corpo, além de pacientes da terceira idade debilitados em leitos de hospitais ou em casos de negligência ${ }^{14-21}$. Já casos de presença de Fannia sp. indicam um avanço no prognóstico para miíase secundária, quando o material orgânico entra em estado de decomposição.

Para melhor compreensão e conhecimento da biodiversidade e epidemiologia desta parasitose, este trabalho objetivou identificar as diferentes espécies e instares de larvais de dípteros muscoides causadores de miíases que foram retiradas de pacientes atendidos em diversos hospitais e clínicas da família do município de Nova Iguaçu, Estado do Rio de Janeiro, no período de janeiro de 2017 a dezembro de 2018.

\section{Material e métodos}

As larvas foram coletadas com o auxílio de pinças pela equipe de enfermagem de Clínicas da Saúde da Família (CSF) e Hospitais Municipais (HM) de Nova Iguaçu em pacientes de atendimento ambulatorial, entre janeiro de 2017 e dezembro de 2018 em pacientes de casos de mí́ases confirmados.

Os espécimes foram coletados diretamente das lesões, sendo sacrificados em éter, lavados em $\mathrm{NaCl}$ a $0,9 \%$ e conservados em recipientes contendo etanol $70 \%$, identificados de acordo com cada caso de miíase e posteriormente foram encaminhados ao Laboratório de Entomologia Médica e Forense (LEMEF) do Instituto Oswaldo Cruz - IOC/FIOCRUZ, para realização das identificações, bem como o fornecimento de informações sobre os casos para o banco de dados do laboratório.

No laboratório, as larvas foram transferidas para $\mathrm{KOH}$ a $10 \%$ e em seguida para o lactofenol, com o objetivo de clarificar e diafanizar o corpo da larva. Estas eram montadas entre lâmina e lamínula para serem analisadas com o auxílio do microscópio estereoscópico e identificadas com o uso de chaves dicotômicas de Guimarães \& Papavero (1999) ${ }^{1}$ e Serra-Freire \& Mello $(2006)^{3}$. Todas as etapas de processamento de análise do material foram realizadas dentro de cabine de exaustão química e de acordo com as normas de biossegurança da instituição. Posteriormente, foram depositadas na coleção entomológica do LEMEF/IOC (FIOCRUZ).

\section{Resultados}

Das amostras encaminhadas pelos serviços de saúde municipal, foram analisadas e identificadas 
Tabela 1. Número de larvas de acordo com os respectivos instares larvais de Cochliomyia hominivorax, coletadas de miíases humanas provenientes de Clínicas da Saúde da Família do município de Nova Iguaçu, Rio de Janeiro no período de janeiro de 2017 a dezembro de 2018.

\begin{tabular}{cccccc}
\hline Pacientes & $\begin{array}{c}\mathbf{N}^{\circ} \text { de } \\
\text { Larvas } \\
\text { (Total) }\end{array}$ & $\mathbf{1}^{\circ}$ instar & $\mathbf{2}^{\circ}$ instar & $\mathbf{3}^{\circ}$ instar & $\begin{array}{c}\text { Farata } \\
\text { (fase de } \\
\text { transição) }\end{array}$ \\
\hline I & 98 & - & - & 98 & - \\
II & 45 & - & - & 44 & $01\left(2^{\circ} / 3^{\circ}\right)$ \\
III & 34 & - & - & 34 & - \\
IV & 13 & - & - & 13 & - \\
V & 7 & - & 3 & 2 & $02\left(2^{\circ} / 3^{\circ}\right)$ \\
VI & 131 & 13 & 16 & 101 & $01\left(1^{\circ} / 2^{\circ}\right)$ \\
VII & 402 & 94 & 139 & 169 & - \\
Total & $\mathbf{7 3 0}$ & $\mathbf{1 0 7}$ & $\mathbf{1 5 8}$ & $\mathbf{4 6 1}$ & $\mathbf{4}$ \\
\hline
\end{tabular}

Tabela 2. Número de larvas de acordo com os respectivos instares larvais e espécies, coletadas de miíases humanas provenientes de Hospitais Municipais do município de Nova Iguaçu, Estado do Rio de Janeiro no período de janeiro de 2017 a dezembro de 2018.

\begin{tabular}{cccccc}
\hline Pacientes & $\begin{array}{c}\mathbf{N}^{\circ} \text { de } \\
\text { Larvas } \\
\text { (Total) }\end{array}$ & $\mathbf{1}^{\circ}$ instar & $\mathbf{2}^{\circ}$ instar & $\mathbf{3}^{\circ}$ instar & $\begin{array}{c}\text { Farata } \\
\text { (fase de } \\
\text { transição) }\end{array}$ \\
\hline I & 1 & - & - & $1(\mathrm{DH})$ & - \\
II & 1 & $1(\mathrm{CH})$ & - & - & - \\
III & 35 & - & $34(\mathrm{CH})$ & $1(\mathrm{CH})$ & - \\
IV & 6 & - & $6(\mathrm{CH})$ & - & - \\
V & 3 & $3(\mathrm{CH})$ & - & - & - \\
VI & 87 & - & - & $87(\mathrm{CH})$ & - \\
VII & 4 & - & - & $4(\mathrm{CH})$ & - \\
VIII & 13 & 01 & - & $12(\mathrm{CH})$ & - \\
& & $(\mathrm{FN})$ & & & - \\
Total & $\mathbf{1 5 0}$ & $\mathbf{5}$ & $\mathbf{4 0}$ & $\mathbf{1 0 5}$ & - \\
\hline
\end{tabular}




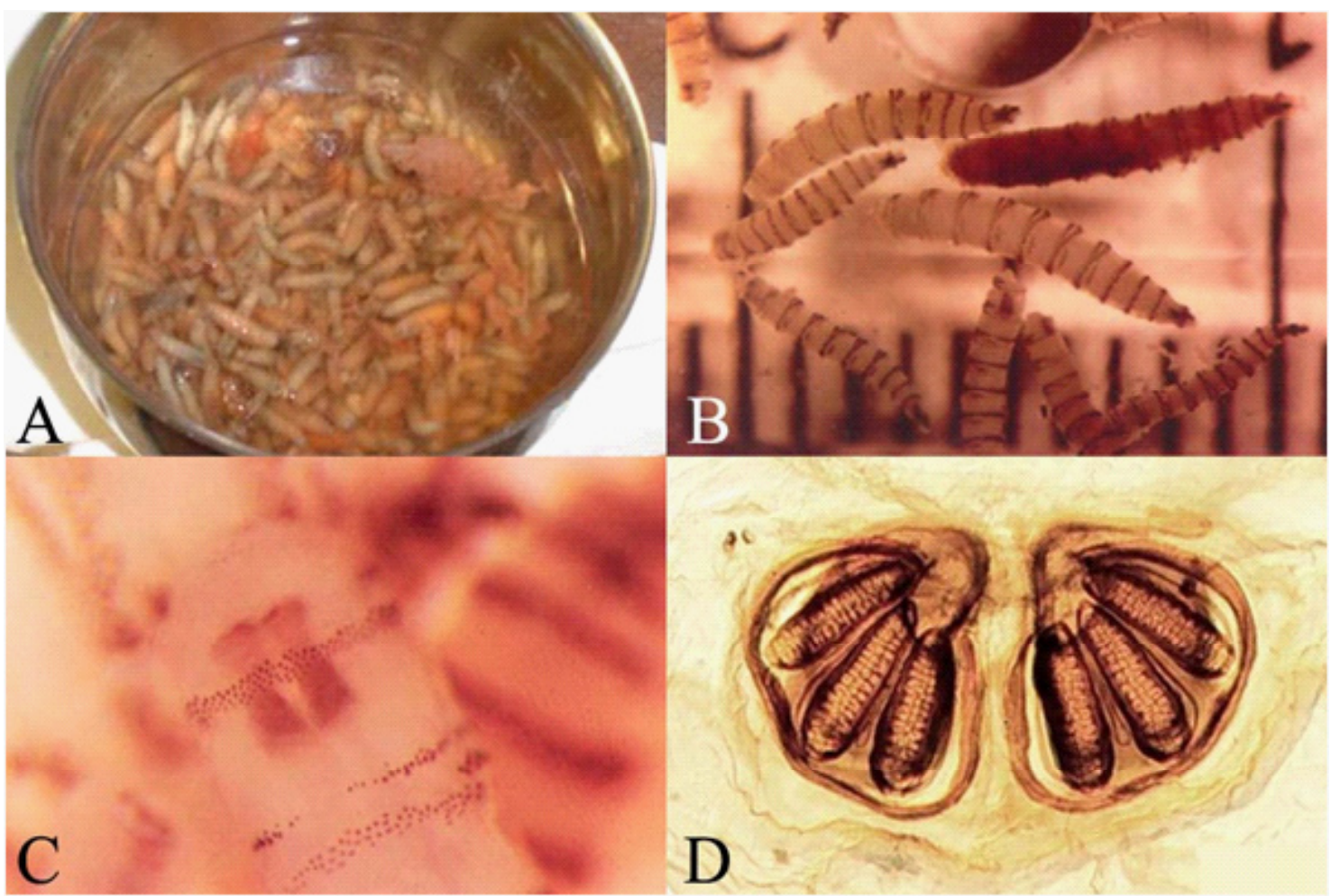

Figura 1. Cochliomyia hominivorax (A) Larvas de $3^{\circ}$ instar; (B) Larvas de $3^{\circ}$ instar montadas entre lâmina e lamínula; (C) Porção final da larva de $3^{\circ}$ instar mostrando as traqueias respiratórias; (D) Peritrema mostrando os estigmas respiratórios da larva de $3^{\circ}$ instar, coletadas de míáases humanas provenientes de Clínicas da Saúde da Família e de Hospitais Municipais do município de Nova Iguaçu, Rio de Janeiro no período de janeiro de 2017 a dezembro de 2018.

Fonte: Acervo do LEMEF-IOC/FIOCUZ

um total de 880 larvas provenientes de 15 pacientes diagnosticados com miĺase. Deste total, 878 foram identificadas como $C$. hominivorax (Figura 1), uma como D. hominis e uma como Fannia sp.

As CSF contabilizaram um maior número de larvas coletadas, totalizando 730 espécimes de sete pacientes (Tabela 1). Todos os espécimes identificados das CSF pertencem à espécie C. hominivorax. Quanto à relação de número de larvas por paciente, a variação encontrada é bem alta, a tabela 1 mostra que essa quantidade varia de 402 larvas, encontradas no paciente VII, e sete larvas, encontradas no paciente V.

Quando analisadas de acordo, com os instares larvais encontrados é possível observar que o terceiro instar foi o mais abundante, totalizando 461 larvas neste estágio $(63,2 \%$ do total), seguido por larvas de segundo instar com 158 indivíduos $(21,6 \%)$, e por larvas de primeiro instar com 107 espécimes, ou 14,7\%. Larvas faratas só foram encontradas nas amostras coletadas nas CSF, em um total de quatro espécimes $(0,5 \%)$, sendo um indivíduo do primeiro para segundo instar e três indivíduos em transição do segundo para o terceiro instar.

Nos HM foram totalizadas 150 larvas coletadas em oito pacientes (Tabela 2). Destas, 148 pertenciam à espécie $C$. hominivorax e foram nestes locais onde foram coletados os exemplares de D. hominis e Fannia sp. O paciente I, que foi diagnosticado com míase furuncular causada por $D$. hominis só apresentou este único indivíduo. Já o paciente VIII, apresentou colonização pelas espécies $C$. hominivorax em associação com um exemplar da espécie Fannia sp., conforme exposto na tabela 2.

As larvas identificadas, em sua maioria, estavam no terceiro instar larval, com 105 espécimes $(70 \%$ do total de larvas coletados), seguido por larvas de segundo instar, com 40 indivíduos $(26,7 \%)$ e apenas cinco larvas $(3,3 \%)$ foram encontradas no estágio inicial, no primeiro instar. Destas últimas podemos citar o espécime de Fannia sp.

Analisando a prevalência das espécies encontradas em ambos os serviços de saúde, nota-se que $C$. hominivorax apresentou a maior porcentagem com $99,8 \%$ de todos os casos analisados (Figura 2). Já D. hominis e Fannia sp. obtiveram porcentagens iguais, com $0,1 \%$ de prevalência cada.

\section{Discussão}

Nos casos relatados na CSF e em HM a maioria das espécies encontradas foi de $C$. hominivorax em 
avançado estágio de desenvolvimento do imaturo $\left(2^{\circ}\right.$ e $3^{\circ}$ instares), indicando casos de mí́ases primárias com demora na procura médica em busca de tratamento adequado, enquanto poucas larvas foram encontradas no $1^{\circ}$ instar.

Segundo Gomez et al. (2003) ${ }^{14}$ e Moya-Borja $(2003)^{22}$, as larvas eclodem do ovo após 12-24 horas em contato com o corpo do mamífero e completam seu ciclo em um período que pode variar de cinco a sete dias, dependendo das condições abióticas em que se encontram, abandonando o local de desenvolvimento após concluírem o estágio L3 e empupando, para assim continuar o ciclo de vida.

O paciente VIII (caso do HM) apresentou colonização por espécimes de $C$. hominivorax no último estágio de desenvolvimento ( $3^{\circ}$ instar), além da presença de um indivíduo de Fannia sp., o que aponta para uma demora na busca do tratamento e início de uma possível necrose no local do ferimento. Caso necrosado, o odor da ferida pode ter atraído espécies de hábitos necrófagos, ocasionando a postura de ovos e posterior desenvolvimento do imaturo na ferida, indicando a probabilidade da presença de uma míáase primária em consonância com uma míase secundária.

Miíase é uma zoodermatose comum em zonas rurais e urbanas, especialmente na periferia, apresentando importância para a Saúde Pública por conta do forte componente social relacionada ao seu aparecimento, estando relacionada à pobreza e à falta de cuidados básicos de higiene e saúde ${ }^{17}$. Os casos de míases ocorrem, de forma geral, em indivíduos com baixo nível de instrução, com pouca higiene corporal e oral e/ou acesso limitado a saneamento básico e serviços de saúde, além de pacientes com distúrbios psiquiátricos, imunodeprimidos, diabéticos ou etilistas ${ }^{17}$.

Segundo Bernhardt et al. (2019) $)^{23}$, ao fazer levantamento bibliográfico de publicações entre 1997 e 2017, casos de mí́ases têm sido reportados no mundo todo: Europa, Ásia, África, América do Sul e Caribe. Na América e no Caribe foram relatados 208 casos, sendo que 104 destes ocorreram na América do Sul, 65 na América Central, 37 na América do Norte (incluindo Canadá) e dois casos no Caribe. Destes 208 casos, $155(74,5 \%)$ foram causados por $C$. hominivorax e D. hominis nas Américas do Sul e Central.

No estudo de Nascimento et al. (2005) ${ }^{24}$ os autores analisaram casos de miíases humanas por $C$. hominivorax em hospitais públicos de Recife, encontrando poucas ocorrências $(n=24)$ entre novembro de 1999 e outubro de 2002. Porém, os autores acreditam que casos de míases, diferentemente do que é comumente expressado na literatura, não sejam de ocorrência rara. Tais autores acreditam que os casos sejam inicialmente minimizados e subestimados, justamente por conta da população que geralmente é afetada por esta enfermidade, o que pode, inclusive, levar a uma procura tardia por ajuda médica ou tratamento pelo paciente, como observado no presente estudo por conta das larvas em estágio avançado de desenvolvimento de $C$. hominivorax, além da presença de Fannia sp. em estágio inicial ( $1^{\circ}$ instar).

Fernandes et al. (2009) ${ }^{25}$ realizaram um estudo no Hospital das Clínicas da Universidade Federal de Goiás, com dados entre fevereiro de 2005 e agosto de 2006, com sessenta e seis (66) casos notificados. Os autores observaram que as principais causas para as subnotificações dos casos são a não obrigatoriedade do registro, a repulsa por parte dos pacientes e dos profissionais de saúde e o desconhecimento desta condição.

Tais fatores levam ao descarte inadequado das larvas, que poderiam ser conduzidas para os laboratórios parceiros para identificação correta da espécie e registro dos dados, inclusive para a realização de estudos epidemiológicos que pudessem elaborar relatórios detalhados sobre as mí́ases em cada região do país e estes dados serem encaminhados ao Ministério da Saúde e desta forma, além de possuir tais dados, poder traçar medidas de controle desta parasitose considerada negligenciada.

Ainda segundo Nascimento et al. (2005) ${ }^{24}$, os autores acreditam que a maioria dos casos, e possivelmente os mais simples de tratar, recebam atendimento primeiramente em postos de saúde da família, com os casos mais complexos sendo encaminhados para hospitais. A maioria dos casos de miĺases diagnosticados no presente estudo ocorreu justamente nas CSF, com a menor quantidade dos casos relatados nos HM de Nova Iguaçu.

Justamente os casos presentes nas CSF podem ter sido ali diagnosticados por conta da facilidade de acesso dos pacientes ao local, porém com relação à gravidade do caso, a maioria destes apresentava-se em estágio avançado por conta do rápido crescimento das larvas. O caso que chama mais a atenção é precisamente o encontrado em HM com míáase secundária por conta da presença do espécime de Fannia sp., demonstrando um possível agravo no quadro clínico.

Além dos diversos fatores discutidos anteriormente, que de forma geral influenciam para a ocorrência de miíases em uma determinada população com perfil socioeconômico baixo, o desconhecimento da condição pela população pode ser um fator de agravamento de casos simples, podendo desta forma dificultar um tratamento pela equipe médica.

Atendimentos em unidades públicas de saúde, como hospitais municipais e clínicas da saúde da família, devem sempre notificar os casos encontrados e procurar parcerias com laboratórios de pesquisa, a fim de melhores esclarecimentos para o paciente e a população na própria unidade, conscientizando e alertando a mesma de tais infestações e de suas consequências. 


\section{Conclusões}

Foi encontrada uma predominância da espécie $C$. hominivorax nos casos de miíase, além da presença de outras espécies como $D$. hominis e Fannia sp., havendo uma maior incidência de larvas de $3^{\circ}$ instar sem a presença de larvas faratas.

Este estudo procurou proporcionar um melhor entendimento de como as míases são tratadas, além da necessidade de aproximação de grupos de pesquisa e profissionais de saúde com os cidadãos, alertando dos perigos e complicações desta afecção.

Existe ainda uma grande necessidade de conhecimento dos casos que ocorrem em demais municípios do estado do Rio de Janeiro, bem como para todos os estados (e seus municípios) do país, para que melhores medidas de conscientização da população sejam elaboradas e aplicadas.

\section{Agradecimentos}

Os autores agradecem as Clínicas de Saúde da Família e os Hospitais Municipais de Nova Iguaçu, seus profissionais de saúde pela colaboração e doação dos espécimes avaliados no laboratório, além das instituições de fomento.

\section{Fonte de financiamento}

Ações Estratégicas para o Desenvolvimento e Fortalecimento dos Laboratórios Credenciados e das Áreas de Apoio à Pesquisa do Instituto Oswaldo Cruz (PAEF-IOC/FIOTEC), Coordenação de Aperfeiçoamento de Pessoal de Nível Superior(CAPES), Conselho Nacional de Pesquisa e Desenvolvimento Tecnológico (CNPq) e Fundação Carlos Chagas Filho de Amparo à Pesquisa do Estado do Rio de Janeiro (FAPERJ).

\section{Referências bibliográficas}

1. Guimarães JH, Papavero N. Myiasis in man and animals in the Neotropical region, bibliographic database. São Paulo: Plêiade/FAPESP; 1999. 308 p.

2. Rey L. Parasitologia: Parasitos e Doenças Parasitárias do Homem nas Américas e na África. Rio de Janeiro: Editora Guanabara Koogan; 2001. 856 $\mathrm{p}$

3. Serra-Freire NM, de Mello RP. Entomologia \& Acarologia na Medicina Veterinária. 1o ed. Rio de Janeiro: L.F. Livros; 2006. 200 p.

4. Thomas DB, Mangan, RL. Oviposition and Wound-Visiting Behavior of the Screwworm Fly, Cochliomyia hominivorax (Diptera: Calliphoridae). Ann Entomol Soc Am. 1989;82(4):526-34.

5. Gomes A, Honer MR, Koller WW, da Silva RL. Vetores de ovos de Dermatobia hominis (L. JR. 1781) (Diptera; Cuterebridae) na região de Cerrados do Mato Grosso do Sul, Brasil. Rev Bras Parasitol Vet. 1998;7(1):3740 .

6. Leite R, Rodríguez Z, Faccini J, Oliveira P, Fernandes A. First Report of Haematobia irritans (L.) (Diptera: Muscidae) as Vector of Dermatobia hominis
(L.jr.) (Diptera: Cuterebridae) in Minas Gerais, Brazil. Mem I Oswaldo Cruz. 1998;93(6):761-2.

7. Aguiar GB, Dias AS, Sobreira RR, Melotti VD. Prevalência de Dermatobia hominis em bovinos abatidos no município de Anchieta, Espírito Santo. Ciência Animal. 2018;28(2):50-8.

8. Chillcott JG. A Revision of the Nearctic Species of Fanniinae (Diptera: Muscidae). Mem Entomol Soc Can. 1960;92(S14):5-295.

9. Holloway BA. Larvae of New Zealand Fanniidae (Diptera: Calyptrata). New Zeal J Zool. 1984;11(3):239-57.

10. Gomes PR, Koller WW, Gomes A, Carvalho CJB, Zorzatto JR. Dípteros fanídeos vetores de ovos de Dermatobia hominis em Campo Grande, Mato Grosso do Sul. Pesq Vet Brasil. 2002;22(3):114-8.

11 Espindola CB, Couri MS. Fannia flavicincta Stein (Diptera, Fanniidae): a new vector of Dermatobia hominis (Linnaeus Jr.) (Diptera, Cuterebridae). Rev Bras Zool. 2004;21(1):115-6.

12. Maia BMC, Prado AM, Purgato NCS, Mira CT, Thyssen PJ. Registro de forésia de ovos de Dermatobia hominis (L.) (Diptera, Oestridae) por Fannia canicularis (L.) e Fannia punctipennis (Albuquerque) (Diptera, Fanniidae) em São Paulo, Brasil. EntomoBrasilis. 2018;11(3):226-9.

13. Ribeiro FAQ, Pereira CSB, Alves A, Marcon MA. Tratamento de mí́ase humana cavitária com ivermectina oral. Rev. Bras. Otorrinolaringol 2001;67(6):755-61

14. Gomez RS, Perdigão PF, Pimenta FJGS, Rios Leite AC, Tanos de Lacerda JC, Custódio Neto AL. Oral myiasis by screwworm Cochliomyia hominivorax. Br J Oral Maxillofac Surg. 2003;41(2):115-6.

15. Martinez CAR, Romani G, Priolli DG, Campos AA de, Carneiro V de PP, Dalbem CAG. Miíase vulvar: relato de caso. Rev Bras Ginecol Obstet. $2003 ; 25(4): 291-5$.

16. Fares NH, de Melo DV, Stucchi N, Carvalhosa AA, Castro PHS, Siqueira CRB. Míase em paciente com 10 anos de idade: relato de caso clínico e revisão de literatura. Rev de Clín Pesq Odontol. 2005;1(4):49-54.

17. Marquez AT, Mattos M da S, Nascimento SB. Mí́ases associadas com alguns fatores sócio-econômicos em cinco áreas urbanas do Estado do Rio de Janeiro. Rev Soc Bras Med Tro. 2007;40(2):175-80.

18. Ferraz ACP, Nunes RV, Gadelha BQ, Nascimento BP, de Barros PREM, Coelho VMA, Lessa CSS. Raro caso de miĺases por Cochliomyia hominivorax (Diptera: Calliphoridae) e Dermatobia hominis (Diptera: Oestridae) em paciente humano. Arq Ciênc Saúde. 2008;15(3):142-4.

19. Martins Junior JC, Keim FS, Iarocrinski J. Oftalmomiíase pós-traumática: relato de caso e revisão de literatura. Rev Bras Oftalmol. 2010;69(4):264-8.

20. Valviesse VRGA, Ferraz ACP, Proença B, Werneck GRN, Aguiar VM, Lessa CSS. Míiase com exposição de calota craniana causada pela associação de Cochliomyia hominivorax (Coquerel, 1858), Cochliomyia macellaria (Fabricius, 1775) e Chrysomya albiceps (Wiedemann, 1819), (Diptera: Calliphoridae) em um paciente atendido em Hospital Público, Rio de Janeiro. Entomotropica. 2014;29(3):191-6.

21. Rodrigues FT, Klemig LR, Cardozo MRP, Alves PC, Aguiar VM, Lessa CS. Myiasis associated with an invasive ductal carcinoma of the left breast: case study. Rev Ins Med Trop São Paulo. 2017;59(0):1-6.

22. Moya Borja GE. Erradicação ou manejo integrado das miíases neotropicais das Américas? Pesq Vet Brasil. 2003;23(3):131-8.

23. Bernhardt V, Finkelmeier F, Verhoff MA, Amendt J. Myiasis in humans-a global case reporte valuation and literature analysis. Parasitol Res. 2019;118(2):389-97.

24. Nascimento EMF do, Oliveira JB de, Paes MJ, Lobo A de P, Silva ALA da, Santos Júnior ER dos, et al. Mí́ases humanas por Cochliomyia hominivorax (Coquerel, 1858) (Diptera, Calliphoridae) em hospitais públicos na cidade do Recife, Pernambuco, Brasil. Entomología y Vectores. 2005;12(1):37-51.

25. Fernandes LF, Pimenta FC, Fernandes FF. First Report of Human Myiasis in Goiás State, Brazil: Frequency of Different Types of Myiasis, Their Various Etiological Agents, and Associated Factors. J Parasitol. 2009;95(1):32-8. 